\title{
REMARKS ON THE CARTAN FORMULA AND ITS APPLICATIONS*
}

\author{
KEFENG LIU $^{\dagger}$ AND SHENG RAO $\ddagger$
}

\begin{abstract}
In this short note, we present certain generalized versions of the commutator formulas of some natural operators on manifolds, and give some applications.
\end{abstract}

Key words. Deformation theory, Kodaira-Spencer theory.

AMS subject classifications. 23G05, 14D15.

1. Introduction. The purpose of this note is to present several general commutator formulas of certain natural operators on Riemannian manifolds, complex manifolds and generalized complex manifolds. We would like to point out that such commutator formulas are essentially consequences of the classical Cartan formula for Lie derivative, but they have deep applications in geometry such as in studying the smoothness of deformation spaces of manifolds. For example one direct consequence of the commutator formula is the Tian-Todorov lemma which is essential for proving the smoothness of the deformation space of Calabi-Yau manifolds in $[10,11]$ and also [12]. The general commutator formulas derived in the note also have applications in proving smoothness of more general deformation spaces such as that of the generalized complex manifolds in [6]. We will discuss the applications of these commutator formulas in deformation theory in our subsequent work.

2. Cartan formula and a general commutator formula. In this section we will present a general commutator formula on a Riemannian manifold. We first fix notations. Given a smooth vector field $X$, a smooth vector bundle $V$ on the smooth Riemannian manifold $M$, and a connection $\nabla$ on $V$ which extends to covariant derivative on the space of smooth $V$-valued differential forms $\Omega^{*}(V)$, we denote by $L_{X}$ the Lie derivative acting on $\Omega^{*}(V)$ and by $\lrcorner$ the contraction operator. We will also denote by $\iota_{X}$ the contraction of a differential form by the vector field $X$. [., $\left.\cdot\right]$ will always denote the usual Lie bracket.

Our starting point is the following formula as Cartan observed

$$
\left.\left.L_{X} \omega=X\right\lrcorner(\nabla \omega)+\nabla(X\lrcorner \omega\right), \text { for } \omega \in \Omega^{*}(V) .
$$

Then our general commutator formula can be stated as follows.

LEMma 2.1. For any two smooth vector fields $X$ and $Y$ on $M$, there holds

$$
[X, Y]\lrcorner \omega=X\lrcorner \nabla(Y\lrcorner \omega)+\nabla(X\lrcorner(Y\lrcorner \omega))-Y\lrcorner(X\lrcorner \nabla \omega)-Y\lrcorner \nabla(X\lrcorner \omega) .
$$

Proof. On one hand, it is obvious by Cartan's formula (2.1) that

$$
\left.\left.\left.\left.\left.L_{X}(Y\lrcorner \omega\right)=X\right\lrcorner \nabla(Y\lrcorner \omega\right)+\nabla(X\lrcorner(Y\lrcorner \omega\right)\right) .
$$

\footnotetext{
*Received November 9, 2010; accepted for publication March 31, 2011.

${ }^{\dagger}$ Department of Mathematics, University of California at Los Angeles, Angeles, CA 90095-1555, USA (liu@math.ucla.edu); and Center of Mathematical Sciences, Zhejiang University, Hangzhou, China (liu@cms.zju.edu.cn).

$\ddagger$ Corresponding author. Center of Mathematical Sciences, Zhejiang University, Hangzhou 310027, China (likeanyone@zju.edu.cn).
} 
And on the other hand,

$$
\left.\left.\left.\left.\left.\left.\left.L_{X}(Y\lrcorner \omega\right)=\left(L_{X} Y\right)\right\lrcorner \omega+Y\right\lrcorner\left(L_{X} \omega\right)=[X, Y]\right\lrcorner \omega+Y\right\lrcorner(X\lrcorner \nabla \omega+\nabla(X\lrcorner \omega\right)\right),
$$

where the last identity applies Cartan's formula (2.1) again. Then (2.2) follows from these two identities above.

REMARK $2.2([2])$. This formula can be considered as a slight generalization of the well-known commutator formula of Lie derivatives acting on differential forms

$$
\left[L_{X}, \iota_{Y}\right]=\iota_{[X, Y]}
$$

It is easy to see that the commutator formula (2.3) is a special case of our formula when $V$ is taken as a trivial bundle on the manifold $M$. In fact, applying both sides of (2.2) to a differential form $\tau \in \Omega^{*}(M)$, we easily get

$$
Y\lrcorner(X\lrcorner d \tau)=X\lrcorner d(Y\lrcorner \tau)+d(X\lrcorner(Y\lrcorner \tau))-Y\lrcorner d(X\lrcorner \tau)-[X, Y]\lrcorner \tau,
$$

which can also be written as

$$
\left.\left.[X, Y]\lrcorner \tau=L_{X}(Y\lrcorner \tau\right)-Y\right\lrcorner\left(L_{X} \tau\right),
$$

and is just the formula (2.3). (See Formula LIE 5 of Proposition 5.3 on pp. 140 of [5].) Furthermore, if $\tau \in \Omega^{1}(M)$, then (2.4) becomes our familiar identity

$$
d \tau(X, Y)=X(\tau(Y))-Y(\tau(X))-\tau([X, Y]),
$$

by the vanishing of $d(X\lrcorner(Y\lrcorner \tau))$ in (2.4).

Another proof of this formula is to use formula for covariant derivative. Without loss of generality, we just consider the special case that $V$ is a line bundle. We denote by $\theta$ and $\tau$ the connection 1-form (matrix) with respect to the connection $\nabla$ and a form of degree $k$ on $M$ respectively. By definition, the covariant derivative of $\tau \otimes s \in \Omega^{k}(V)$ is given by

$$
\nabla(\tau \otimes s)=d \tau \otimes s+(-1)^{k} \tau \wedge \nabla s
$$

where $s$ is a smooth section of $V$. Firstly, we can easily check that

$$
Y\lrcorner(X\lrcorner(\tau \wedge \theta))=(X\lrcorner(Y\lrcorner \tau)) \wedge \theta-X\lrcorner((Y\lrcorner \tau) \wedge \theta)+Y\lrcorner((X\lrcorner \tau) \wedge \theta) .
$$

Actually, a direct calculation shows that

$$
\begin{aligned}
L H S & \left.\left.=Y\lrcorner((X\lrcorner \tau) \wedge \theta+(-1)^{k} \tau \wedge(X\lrcorner \theta\right)\right) \\
& \left.\left.\left.\left.=Y\lrcorner((X\lrcorner \tau) \wedge \theta)+(-1)^{k}(X\lrcorner(\theta \wedge(Y\lrcorner \tau)\right)-(-1) \theta \wedge(X\lrcorner(Y\lrcorner \tau\right)\right)\right) \\
& \left.\left.\left.\left.=Y\lrcorner((X\lrcorner \tau) \wedge \theta)+(-1)^{k}(-1)^{k-1} X\right\lrcorner((Y\lrcorner \tau) \wedge \theta\right)+(-1)^{k+k-2}(X\lrcorner(Y\lrcorner \tau\right)\right) \wedge \theta \\
& =R H S .
\end{aligned}
$$

Without loss of generality, assume that $s$ is a smooth local frame of the smooth line bundle $V$. By adding up the tensor products $(2.7) \otimes(-1)^{k} s$ and $(2.4) \otimes s$, we have reproved our formula $(2.2)$. 
3. Commutator formula on complex manifolds. In this section, we consider an $n$-dimensional complex manifold $M$ and a holomorphic vector bundle $V$ over it. As the applications of our commutator formula, we derive a general commutator identity for any $V$-valued $(n, *)$-form and also any $V$-valued $(*, *)$-form on $M$, and as an easy consequence we derive the Tian-Todorov lemma. $\nabla$ will always denote the Chern connection of the Hermitian holomorphic vector bundle $V$ throughout this section.

Following pp. 152 of [8], we first introduce some notations. As usual, we let $A^{p, q}(V):=A^{p, q}(M, V)$ be the space of smooth $(p, q)$-forms with coefficients in $V$. If $X=\sum_{i=1}^{n} X^{i} \partial_{i}$ and $Y=\sum_{i=1}^{n} Y^{i} \partial_{i}$, then

$$
[X, Y]=\sum_{i, j}\left(X^{i} \partial_{i} Y^{j}-Y^{i} \partial_{i} X^{j}\right) \partial_{j}
$$

For the generalization, let

$$
\varphi^{i}=\frac{1}{p !} \sum \varphi_{\bar{j}_{1}, \cdots, \bar{j}_{p}}^{i} d \bar{z}^{j_{1}} \wedge \cdots \wedge d \bar{z}^{j_{p}}
$$

and

$$
\psi^{i}=\frac{1}{q !} \sum \psi_{\bar{k}_{1}, \cdots, \bar{k}_{q}}^{i} d \bar{z}^{k_{1}} \wedge \cdots \wedge d \bar{z}^{k_{q}}
$$

Definition 3.1. For $\varphi=\sum_{i} \varphi^{i} \otimes \partial_{i}$ and $\psi=\sum_{i} \psi^{i} \otimes \partial_{i}$, we define

$$
[\varphi, \psi]=\sum_{i, j=1}^{n}\left(\varphi^{i} \wedge \partial_{i} \psi^{j}-(-1)^{p q} \psi^{i} \wedge \partial_{i} \varphi^{j}\right) \otimes \partial_{j},
$$

where

$$
\partial_{i} \varphi^{j}=\frac{1}{p !} \sum \partial_{i} \varphi_{\bar{j}_{1}, \cdots, \bar{j}_{p}}^{j} d \bar{z}^{j_{1}} \wedge \cdots \wedge d \bar{z}^{j_{p}}
$$

and similarly for $\partial_{i} \psi^{j}$. In particular, if $\varphi, \psi \in A^{0,1}\left(M, T_{M}^{1,0}\right)$, then

$$
[\varphi, \psi]=\sum_{i, j=1}^{n}\left(\varphi^{i} \wedge \partial_{i} \psi^{j}+\psi^{i} \wedge \partial_{i} \varphi^{j}\right) \otimes \partial_{j} .
$$

Proposition 3.2. For any holomorphic vector bundle $V$, any $\omega \otimes s \in A^{n, *}(V)^{1}$ and any $\phi_{i} \in A^{0,1}\left(M, T_{M}^{1,0}\right), i=1,2$, there holds

(3.1) $\left.\left.\left.\left.\left.\left.\left.\left[\phi_{1}, \phi_{2}\right]\right\lrcorner(\omega \otimes s)=\left(\phi_{1}\right\lrcorner \partial\left(\phi_{2}\right\lrcorner \omega\right)\right) \otimes s-\partial\left(\phi_{2}\right\lrcorner\left(\phi_{1}\right\lrcorner \omega\right)\right) \otimes s+\left(\phi_{2}\right\lrcorner \partial\left(\phi_{1}\right\lrcorner \omega\right)\right) \otimes s$, or equivalently,

$$
\left.\left.\left.\left.\left.\left.\left.\left[\phi_{1}, \phi_{2}\right]\right\lrcorner \omega=\phi_{1}\right\lrcorner \partial\left(\phi_{2}\right\lrcorner \omega\right)-\partial\left(\phi_{2}\right\lrcorner\left(\phi_{1}\right\lrcorner \omega\right)\right)+\phi_{2}\right\lrcorner \partial\left(\phi_{1}\right\lrcorner \omega\right) .
$$

Proof. We first show the following identity

$[X, Y]\lrcorner(\tau \otimes s)=(X\lrcorner d(Y\lrcorner \tau)) \otimes s+d(X\lrcorner(Y\lrcorner \tau)) \otimes s-(Y\lrcorner(X\lrcorner d \tau)) \otimes s-(Y\lrcorner d(X\lrcorner \tau)) \otimes s$,

\footnotetext{
${ }^{1}$ Here $\omega \in A^{n, *}(M)$ and $s$ is a smooth section of $V$. In the following, we will adopt this convention without chance for confusion.
} 
for $\tau \otimes s \in A^{n, *}(V)$.

One way to approach this identity is a direct application of (2.4), while here we adopt a lengthy but more intrinsic proof. In fact, we have

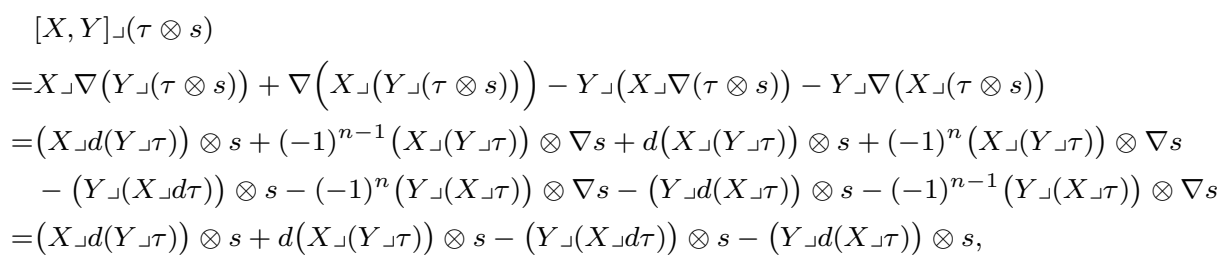

where the first equality applies our general commutator formula (2.2).

Then, if we take $\tau$ as $\omega \wedge d \bar{z}^{k_{1}} \wedge d \bar{z}^{k_{2}}$ and $X, Y$ as $\left(\phi_{1}\right)_{\bar{k}_{1}},\left(\phi_{2}\right)_{\bar{k}_{2}}$, respectively, we can conclude the proof of (3.1). In fact, set

$$
\phi_{i}=\left(\phi_{i}\right)_{\bar{k}} \otimes d \bar{z}^{k},
$$

where $\left(\phi_{i}\right)_{\bar{k}}=\sum_{j=1}^{n}\left(\phi_{i}\right)_{\bar{k}}^{j} \frac{\partial}{\partial z^{j}}$ is a vector field of type $(1,0)$. Then by definition it is easy to check that

$$
\left[\phi_{1}, \phi_{2}\right]=\left[\left(\phi_{1}\right)_{\bar{k}_{1}},\left(\phi_{2}\right)_{\bar{k}_{2}}\right] \otimes\left(d \bar{z}^{k_{1}} \wedge d \bar{z}^{k_{2}}\right)
$$

and thus

$$
\left.\left.\left.\left[\phi_{1}, \phi_{2}\right]\right\lrcorner \omega=\left(\left[\left(\phi_{1}\right)_{\bar{k}_{1}},\left(\phi_{2}\right)_{\bar{k}_{2}}\right]\right\lrcorner \omega\right) \wedge\left(d \bar{z}^{k_{1}} \wedge d \bar{z}^{k_{2}}\right)=\left[\left(\phi_{1}\right)_{\bar{k}_{1}},\left(\phi_{2}\right)_{\bar{k}_{2}}\right]\right\lrcorner\left(\omega \wedge d \bar{z}^{k_{1}} \wedge d \bar{z}^{k_{2}}\right) .
$$

Hence, by applying our formula (2.2) in the special case (i.e., the commutator formula (2.4) as pointed out in Remark 2.2) to the complex setting, and taking $\tau=\omega \wedge d \bar{z}^{k_{1}} \wedge$ $d \bar{z}^{k_{2}}$ and only the components of $(n-1,2)$-type forms, one has

$$
\begin{aligned}
& {\left.\left[\left(\phi_{1}\right)_{\bar{k}_{1}},\left(\phi_{2}\right)_{\bar{k}_{2}}\right]\right\lrcorner\left(\omega \wedge d \bar{z}^{k_{1}} \wedge d \bar{z}^{k_{2}}\right) } \\
= & \left.\left.\left.\left.\left(\phi_{1}\right)_{\bar{k}_{1}}\right\lrcorner \partial\left(\left(\phi_{2}\right)_{\bar{k}_{2}}\right\lrcorner\left(\omega \wedge d \bar{z}^{k_{1}} \wedge d \bar{z}^{k_{2}}\right)\right)+\partial\left(\left(\phi_{1}\right)_{\bar{k}_{1}}\right\lrcorner\left(\left(\phi_{2}\right)_{\bar{k}_{2}}\right\lrcorner\left(\omega \wedge d \bar{z}^{k_{1}} \wedge d \bar{z}^{k_{2}}\right)\right)\right) \\
- & \left.\left.\left.\left.\left(\phi_{2}\right)_{\bar{k}_{2}}\right\lrcorner \partial\left(\left(\phi_{1}\right)_{\bar{k}_{1}}\right\lrcorner\left(\omega \wedge d \bar{z}^{k_{1}} \wedge d \bar{z}^{k_{2}}\right)\right)-\left(\phi_{2}\right)_{\bar{k}_{2}}\right\lrcorner\left(\left(\phi_{1}\right)_{\bar{k}_{1}}\right\lrcorner \partial\left(\omega \wedge d \bar{z}^{k_{1}} \wedge d \bar{z}^{k_{2}}\right)\right) .
\end{aligned}
$$

A simple calculation implies that

$$
\left.\left.\left.\left.\left.\left.\left.\left[\phi_{1}, \phi_{2}\right]\right\lrcorner \omega=\phi_{1}\right\lrcorner \partial\left(\phi_{2}\right\lrcorner \omega\right)-\partial\left(\phi_{2}\right\lrcorner\left(\phi_{1}\right\lrcorner \omega\right)\right)+\phi_{2}\right\lrcorner \partial\left(\phi_{1}\right\lrcorner \omega\right),
$$

by the vanishing of the last term of right-hand side of (3.3).

It is interesting to write down the following several useful identities from the proof of (3.2) and (3.1): for any $\omega \otimes s \in A^{*, *}(V)$,

$$
\left.\left.\left.\left.\left.\left.\left.\left.\phi_{1}\right\lrcorner \bar{\partial}\left(\phi_{2}\right\lrcorner \omega\right)-\bar{\partial}\left(\phi_{2}\right\lrcorner\left(\phi_{1}\right\lrcorner \omega\right)\right)+\phi_{2}\right\lrcorner \bar{\partial}\left(\phi_{1}\right\lrcorner \omega\right)-\phi_{2}\right\lrcorner\left(\phi_{1}\right\lrcorner \bar{\partial} \omega\right)=0,
$$

or equivalently,

$$
\left.\left.\left.\left.\left.\left.\left.\left.\left(\phi_{1}\right\lrcorner \bar{\partial}\left(\phi_{2}\right\lrcorner \omega\right)\right) \otimes s-\bar{\partial}\left(\phi_{2}\right\lrcorner\left(\phi_{1}\right\lrcorner \omega\right)\right) \otimes s+\left(\phi_{2}\right\lrcorner \bar{\partial}\left(\phi_{1}\right\lrcorner \omega\right)\right) \otimes s-\left(\phi_{2}\right\lrcorner\left(\phi_{1}\right\lrcorner \bar{\partial} \omega\right)\right) \otimes s=0,
$$

and the (more) complete general commutator formula

$$
\left.\left.\left.\left.\left.\left.\left.\left.\left.\left[\phi_{1}, \phi_{2}\right]\right\lrcorner \omega=\phi_{1}\right\lrcorner \partial\left(\phi_{2}\right\lrcorner \omega\right)-\partial\left(\phi_{2}\right\lrcorner\left(\phi_{1}\right\lrcorner \omega\right)\right)+\phi_{2}\right\lrcorner \partial\left(\phi_{1}\right\lrcorner \omega\right)-\phi_{2}\right\lrcorner\left(\phi_{1}\right\lrcorner \partial \omega\right),
$$


or equivalently,

$$
\begin{aligned}
{\left.\left[\phi_{1}, \phi_{2}\right]\right\lrcorner(\omega \otimes s)=} & \left.\left.\left.\left.\left(\phi_{1}\right\lrcorner \partial\left(\phi_{2}\right\lrcorner \omega\right)\right) \otimes s-\partial\left(\phi_{2}\right\lrcorner\left(\phi_{1}\right\lrcorner \omega\right)\right) \otimes s \\
& \left.\left.\left.\left.+\left(\phi_{2}\right\lrcorner \partial\left(\phi_{1}\right\lrcorner \omega\right)\right) \otimes s-\phi_{2}\right\lrcorner\left(\phi_{1}\right\lrcorner \partial \omega\right) \otimes s .
\end{aligned}
$$

Moreover, there holds

$$
\left.\left.\left.\left.\left.\left.\left.\left.\left.\left[\phi_{1}, \phi_{2}\right]\right\lrcorner \omega=\phi_{1}\right\lrcorner d\left(\phi_{2}\right\lrcorner \omega\right)-d\left(\phi_{2}\right\lrcorner\left(\phi_{1}\right\lrcorner \omega\right)\right)+\phi_{2}\right\lrcorner d\left(\phi_{1}\right\lrcorner \omega\right)-\phi_{2}\right\lrcorner\left(\phi_{1}\right\lrcorner d \omega\right) .
$$

or equivalently,

$$
\begin{aligned}
{\left.\left[\phi_{1}, \phi_{2}\right]\right\lrcorner(\omega \otimes s)=} & \left.\left.\left.\left.\left(\phi_{1}\right\lrcorner d\left(\phi_{2}\right\lrcorner \omega\right)\right) \otimes s-d\left(\phi_{2}\right\lrcorner\left(\phi_{1}\right\lrcorner \omega\right)\right) \otimes s \\
& \left.\left.\left.\left.+\left(\phi_{2}\right\lrcorner d\left(\phi_{1}\right\lrcorner \omega\right)\right) \otimes s-\phi_{2}\right\lrcorner\left(\phi_{1}\right\lrcorner d \omega\right) \otimes s .
\end{aligned}
$$

Based on the argument above, we obtain another general commutator identity for Hermitian holomorphic vector bundle valued $(n, *)$-forms on complex manifolds.

TheOREM 3.3. For any Hermitian holomorphic vector bundle $V$, any $\eta \in$ $A^{n, *}(V)$ and any $\phi_{i} \in A^{0,1}\left(M, T_{M}^{1,0}\right), i=1,2$, one has

$$
\left.\left.\left.\left.\left.\left.\left.\left.\left.\left[\phi_{1}, \phi_{2}\right]\right\lrcorner \eta=\phi_{1}\right\lrcorner \nabla\left(\phi_{2}\right\lrcorner \eta\right)-\nabla\left(\phi_{2}\right\lrcorner\left(\phi_{1}\right\lrcorner \eta\right)\right)+\phi_{2}\right\lrcorner \nabla\left(\phi_{1}\right\lrcorner \eta\right)-\phi_{2}\right\lrcorner\left(\phi_{1}\right\lrcorner \nabla \eta\right),
$$

where $\nabla$ is the Chern connection of the Hermitian holomorphic vector bundle V.

Proof. Let $r=\operatorname{rank}(V)$. Assume that $s=\left\{s_{1}, \cdots, s_{r}\right\}$ is a local holomorphic frame of $V$ and that $h=\left(h_{i \bar{j}}\right)=\left(h\left(s_{i}, s_{j}\right)\right)$ is the matrix of the metric of $h$ under $s$. Without loss of generality, we can locally set

$$
\eta=\sum_{i=1}^{r} \omega_{i} \otimes s_{i} \quad \text { and } \quad \nabla s_{i}=\sum_{j=1}^{r} \theta_{i j} \otimes s_{j}
$$

where $\omega_{i} \in A^{n, *}(M)$ and $\theta_{i j}=\sum_{k=1}^{r} \partial h_{i \bar{k}} \cdot h^{\bar{k} j}$ is the connection $(1,0)$-form of $\nabla$ with respect to $s$.

Now we proceed to our proof. Firstly, for any two functions $f$ and $g$ on the complex manifold $M$, letting $f \omega_{i}$ substitute for $\omega$ in (3.2), one has

$$
\left.\left.\left.\left.\left.\left.\left.\left[\phi_{1}, \phi_{2}\right]\right\lrcorner f \omega_{i}=-\partial\left(\phi_{2}\right\lrcorner\left(\phi_{1}\right\lrcorner f \omega_{i}\right)\right)+\phi_{1}\right\lrcorner \partial\left(\phi_{2}\right\lrcorner f \omega_{i}\right)+\phi_{2}\right\lrcorner \partial\left(\phi_{1}\right\lrcorner f \omega_{i}\right) .
$$

So by (3.2) we have

$$
\left.\left.\left.\left.\left.\left.0=-\partial f \wedge\left(\phi_{2}\right\lrcorner\left(\phi_{1}\right\lrcorner \omega_{i}\right)\right)+\phi_{1}\right\lrcorner\left(\partial f \wedge\left(\phi_{2}\right\lrcorner \omega_{i}\right)\right)+\phi_{2}\right\lrcorner\left(\partial f \wedge\left(\phi_{1}\right\lrcorner \omega_{i}\right)\right)
$$

and its equivalent form

$$
\begin{aligned}
0= & \left.\left.\left.\left.-g \cdot \partial f \wedge\left(\phi_{2}\right\lrcorner\left(\phi_{1}\right\lrcorner \omega_{i}\right)\right) \otimes s_{j}+\phi_{1}\right\lrcorner\left(g \cdot \partial f \wedge\left(\phi_{2}\right\lrcorner \omega_{i}\right)\right) \otimes s_{j} \\
& \left.\left.+\phi_{2}\right\lrcorner\left(g \cdot \partial f \wedge\left(\phi_{1}\right\lrcorner \omega_{i}\right)\right) \otimes s_{j} .
\end{aligned}
$$

Next, submitting $\partial h_{i \bar{k}} \cdot h^{\bar{k} j}$ into (3.9) as $g \cdot \partial f$ and taking sums over $k$ and then over $j$, then we obtain

$$
\left.\left.\left.\left.\left.\left.0=-\nabla s_{i} \wedge\left(\phi_{2}\right\lrcorner\left(\phi_{1}\right\lrcorner \omega_{i}\right)\right)+\phi_{1}\right\lrcorner\left(\nabla s_{i} \wedge\left(\phi_{2}\right\lrcorner \omega_{i}\right)\right)+\phi_{2}\right\lrcorner\left(\nabla s_{i} \wedge\left(\phi_{1}\right\lrcorner \omega_{i}\right)\right)
$$

according to (3.8). 
Then, combining (3.10), (3.1) and (3.4) with $\omega$ and $s$ replaced by $\omega_{i}$ and $s_{i}$ respectively, and summing over $i$, we can complete our proof according to the formula (2.6) for covariant derivative and the assumption (3.8).

Corollary 3.4. For any Hermitian holomorphic vector bundle $V$, any $\eta \in$ $A^{*, *}(V)$ and any $\phi_{i} \in A^{0,1}\left(M, T_{M}^{1,0}\right), i=1,2$, there holds

$$
\left.\left.\left.\left.\left.\left.\left.\left.\left.\left[\phi_{1}, \phi_{2}\right]\right\lrcorner \eta=\phi_{1}\right\lrcorner \nabla\left(\phi_{2}\right\lrcorner \eta\right)-\nabla\left(\phi_{2}\right\lrcorner\left(\phi_{1}\right\lrcorner \eta\right)\right)+\phi_{2}\right\lrcorner \nabla\left(\phi_{1}\right\lrcorner \eta\right)-\phi_{2}\right\lrcorner\left(\phi_{1}\right\lrcorner \nabla \eta\right),
$$

where $\nabla$ is the Chern connection of the Hermitian holomorphic vector bundle $V$.

Proof. Based on the identities (3.6) and (3.7), we can obtain (3.11) by the same computation as we use to prove Theorem 3.3 and the details are left to the readers. $\square$

Next, following the paper of S. Barannikov and M. Kontsevich [1], we can present some reformulation of the above results as follows. Let us fix a $(k, l)$-form $\omega \in A^{k, l}(M)$. It induces a linear map ${ }^{2}$

$$
\left.A^{0, q}\left(M, \bigwedge^{p} T_{M}^{1,0}\right) \longrightarrow A^{k-p, q+l}(M)^{3}: \phi \mapsto \phi\right\lrcorner \omega
$$

We define a map $\Delta_{\omega}$ from $\mathbf{t}$ to $A^{*, *}(M)$ by the formula

$$
\left.\Delta_{\omega} \phi:=\partial(\phi\lrcorner \omega\right) \text {. }
$$

Similarly, let us fix a $V$-valued $(k, l)$-form $\eta \in A^{k, l}(V)$. It induces a linear map

$$
\left.A^{0, q}\left(M, \bigwedge^{p} T_{M}^{1,0}\right) \longrightarrow A^{k-p, q+l}(V): \phi \mapsto \phi\right\lrcorner \eta .
$$

Then, a map $\diamond_{\eta}$ from $\mathbf{t}$ to $A^{*, *}(V)$ is defined as the formula

$$
\left.\diamond_{\eta} \phi:=\nabla(\phi\lrcorner \eta\right) .
$$

Here $\mathbf{t}$ is the differential graded Lie algebras given by

$$
\mathbf{t}=\bigoplus_{k} \mathbf{t}^{k}, \quad \mathbf{t}^{k}=\bigoplus_{p+q-1=k} A^{0, q}\left(M, \bigwedge^{p} T_{M}^{1,0}\right),
$$

endowed with the differential $\bar{\partial}$, and the bracket coming from the cup-product on $\bar{\partial}$-forms and the standard Schouten-Nijenhuys bracket on polyvector fields.

Then we can generalize and restate Proposition 3.2, and restate Identity (3.5) and Corollary 3.4 as follows.

Proposition 3.5. (1) For any $\omega \in A^{n, *}(M)$ and any $\phi_{i} \in A^{0, q}\left(M, \bigwedge^{p} T_{M}^{1,0}\right)$, $i=1,2$, there holds

$$
\left.\left.\left.\left[\phi_{1}, \phi_{2}\right]\right\lrcorner \omega=-\Delta_{\omega}\left(\phi_{1} \wedge \phi_{2}\right)+\phi_{2}\right\lrcorner \Delta_{\omega} \phi_{1}+\phi_{1}\right\lrcorner \Delta_{\omega} \phi_{2} .
$$

(2) For any $\omega \in A^{*, *}(M)$ and any $\phi_{i} \in A^{0,1}\left(M, T_{M}^{1,0}\right), i=1,2$, we have

$$
\left.\left.\left.\left.\left[\phi_{1}, \phi_{2}\right]\right\lrcorner \omega=-\left(\Delta_{\omega}\left(\phi_{1} \wedge \phi_{2}\right)-\phi_{2}\right\lrcorner \Delta_{\omega} \phi_{1}-\phi_{1}\right\lrcorner \Delta_{\omega} \phi_{2}+\left(\phi_{1} \wedge \phi_{2}\right)\right\lrcorner \partial\right) .
$$

\footnotetext{
${ }^{2}$ In our manuscript, this map and also the following map $\left.\phi \mapsto \phi\right\lrcorner \eta$ were mistaken as two isomorphisms, which is kindly pointed out by the referee.

${ }^{3}$ For convention, here we set $k \geq p$.
} 
(3) For any $\eta \in A^{*, *}(V)$ and any $\phi_{i} \in A^{0,1}\left(M, T_{M}^{1,0}\right), i=1,2$, one has

$$
\left.\left.\left.\left.\left[\phi_{1}, \phi_{2}\right]\right\lrcorner \eta=-\left(\diamond_{\eta}\left(\phi_{1} \wedge \phi_{2}\right)-\phi_{2}\right\lrcorner \diamond_{\eta} \phi_{1}-\phi_{1}\right\lrcorner \diamond_{\eta} \phi_{2}+\left(\phi_{1} \wedge \phi_{2}\right)\right\lrcorner \nabla\right) .
$$

Finally, we briefly recall how to derive the original Tian-Todorov lemma from the above commutator formulas.

LEMMA 3.6. Let $M$ be an $n$-dimensional complex manifold with a non-vanishing holomorphic $n$-form $\omega_{0}$, which is given in a local coordinate chart $\left(U ; z^{1}, \cdots, z^{n}\right)$ by $\left.\omega_{0}\right|_{U}=d z^{1} \wedge \cdots \wedge d z^{n}$. Then

a) (Lemma 3.1 in [10], or also Section 2 in [2]) For $\omega_{i} \in A^{n-1,1}(M), i=1,2$,

$$
\left.\left[\omega_{1}, \omega_{2}\right]=-\partial\left(\omega_{2}\right\lrcorner \imath^{-1}\left(\omega_{1}\right)\right)+\omega_{1} \wedge \sharp\left(\partial \omega_{2}\right)+\omega_{2} \wedge \sharp\left(\partial \omega_{1}\right),
$$

where $\imath: A^{0, q}\left(M, T_{M}^{1,0}\right) \rightarrow A^{n-1, q}(M)$ is the natural isomorphism by contraction with $\omega_{0}$ and $\sharp$ denotes the obvious map identifying the $(n, q)$-form $\eta \wedge \omega_{0}$ with the $(0, q)$-form $\eta$ by $\omega_{0}$, i.e., $\sharp\left(\eta \wedge \omega_{0}\right)=\eta$.

b) (Lemma 1.2.4 in [11], or also Lemma 64 in [12]) For $\phi_{i} \in A^{0,1}\left(M, T_{M}^{1,0}\right), i=1,2$, with $\left.\partial\left(\phi_{i}\right\lrcorner \omega_{0}\right)=0$,

$$
\left.\left.\left.\left[\phi_{1}, \phi_{2}\right]\right\lrcorner \omega_{0}=-\partial\left(\phi_{2}\right\lrcorner\left(\phi_{1}\right\lrcorner \omega_{0}\right)\right) .
$$

Actually, both (3.12) and (3.13) can be achieved by (3.2). In fact, for each $\omega_{i}$, we have some $\phi_{i} \in A^{0,1}\left(M, T_{M}^{1,0}\right)$ via $\left.\omega_{i}=\omega_{0}\right\lrcorner \phi_{i}$. Then $\left.\left[\omega_{1}, \omega_{2}\right]=\omega_{0}\right\lrcorner\left[\phi_{1}, \phi_{2}\right]$. Here we need a simple commutator rule, that is, for any $\omega \in A^{k, l}(M)$ and $\psi \in A^{0, q}\left(M, \wedge^{p} T_{M}^{1,0}\right)$, one has

$$
\left.\omega\lrcorner \psi=(-1)^{q(k+l-p)} \psi\right\lrcorner \omega .
$$

So by the commutator rule $(3.14)$, we have $\left.\left[\omega_{1}, \omega_{2}\right]=\left[\phi_{1}, \phi_{2}\right]\right\lrcorner \omega_{0}$ and

$$
\left.\left.\left.\left.\left.\left.\omega_{1} \wedge \sharp\left(\partial \omega_{2}\right)=-\partial\left(\omega_{0}\right\lrcorner \phi_{2}\right)\right\lrcorner \phi_{1}=(-1)^{(n+1)+n-1} \phi_{1}\right\lrcorner \partial\left(\phi_{2}\right\lrcorner \omega_{0}\right)=\phi_{1}\right\lrcorner \partial\left(\phi_{2}\right\lrcorner \omega_{0}\right) .
$$

Similarly, $\left.\left.\omega_{2} \wedge \sharp\left(\partial \omega_{1}\right)=\phi_{2}\right\lrcorner \partial\left(\phi_{1}\right\lrcorner \omega_{0}\right)$. It is easy to check that

$$
\left.\left.\left.\left.\left.-\partial\left(\omega_{2}\right\lrcorner \iota^{-1}\left(\omega_{1}\right)\right)=-\partial\left(\phi_{1}\right\lrcorner\left(\phi_{2}\right\lrcorner \omega_{0}\right)\right)=-\partial\left(\phi_{2}\right\lrcorner\left(\phi_{1}\right\lrcorner \omega_{0}\right)\right) .
$$

Therefore, we obtain an equivalent form of Tian's identity,

$$
\left.\left.\left.\left.\left.\left.\left.\left[\phi_{1}, \phi_{2}\right]\right\lrcorner \omega_{0}=-\partial\left(\phi_{2}\right\lrcorner\left(\phi_{1}\right\lrcorner \omega_{0}\right)\right)+\phi_{1}\right\lrcorner \partial\left(\phi_{2}\right\lrcorner \omega_{0}\right)+\phi_{2}\right\lrcorner \partial\left(\phi_{1}\right\lrcorner \omega_{0}\right),
$$

which is just the identity (3.2) with $\omega=\omega_{0}$.

As for Todorov's identity (3.13), we just need notice that the condition $\left.\partial\left(\phi_{i}\right\lrcorner \omega_{0}\right)=$ 0 results in the vanishing of the last two terms in the right-hand side of (3.15).

By this crucial Tian-Todorov lemma, the well-known $\partial \bar{\partial}$-lemma and Kuranishi's construction of power series, Tian [10] and Todorov [11] proved the famous Bogomolov-Tian-Todorov unobstrution theorem. It can be stated roughly as follows. Let $M$ be a Calabi-Yau manifold, where $n=\operatorname{dim} M \geq 3$. Let $\pi: X \rightarrow S$, with central fiber $\pi^{-1}(0)=M$ be the Kuranishi family of $M$, then the Kuranishi space $S$ is a nonsingular complex analytic space and $\operatorname{dim} S=\operatorname{dim} H_{\mathbb{C}}^{1}\left(M, \Theta_{M}\right)=\operatorname{dim} H_{\mathbb{C}}^{1}\left(M, \Omega^{n-1}\right)$, where $\Theta$ is the holomorphic tangent bundle of $M$. 
4. Twisted commutator formula on generalized complex manifolds. In this section, we prove a twisted commutator formula on generalized complex manifolds, reprove Corollary 3.4 for any Hermitian holomorphic vector bundle and obtain a more general commutator formula in Corollary 4.6 as the applications of our twisted commutator formula.

First of all, let us introduce some notations on generalized complex geometry and we refer the readers to $[3,6]$ and the references therein for a more detailed and systematic treatment of generalized complex geometry.

Let $\check{M}$ be a smooth manifold, $T:=T_{\check{M}}$ the tangent bundle of $\check{M}$ and $T^{*}:=T_{\check{M}}^{*}$ its cotangent bundle. In the generalized complex geometry, for any $X, Y \in C^{\infty}(T)$ and $\xi, \eta \in C^{\infty}\left(T^{*}\right), T \oplus T^{*}$ is endowed with a canonical nondegenerate inner product given by

$$
\langle X+\xi, Y+\eta\rangle=\frac{1}{2}\left(\iota_{X}(\eta)+\iota_{Y}(\xi)\right)
$$

and there is an important canonical bracket on $T \oplus T^{*}$, so-called Courant bracket, which is defined by

$$
[X+\xi, Y+\eta]=[X, Y]+L_{X} \eta-L_{Y} \xi-\frac{1}{2} d\left(\iota_{X}(\eta)-\iota_{Y}(\xi)\right)
$$

Here, $[\cdot, \cdot]$ on the right-hand side is the ordinary Lie bracket of vector fields. Note that on vector fields the Courant bracket reduces to the Lie bracket; in other words, if $p r_{1}: T \oplus T^{*} \rightarrow T$ is the natural projection, then

$$
\left.p r_{1}([A, B])=\left[p r_{1}(A), p r_{1}(B)\right]\right),
$$

for any $A, B \in C^{\infty}\left(T \oplus T^{*}\right)$.

A generalized almost complex structure on $\check{M}$ is a smooth section $J$ of the endomorphism bundle $\operatorname{End}\left(T \oplus T^{*}\right)$, which satisfies both symplectic and complex conditions, i.e. $J^{*}=-J$ (equivalently, orthogonal with respect to the canonical inner product (4.1)) and $J^{2}=-1$. We can show that the obstruction to the existence of a generalized almost complex structure is the same as that for an almost complex structure. (See Proposition 4.15 in [3].) Hence it is obvious that (generalized) almost complex structures only exist on the even-dimensional manifolds. Let $E \subset\left(T \oplus T^{*}\right) \otimes \mathbb{C}$ be the $+i$-eigenbundle of the generalized almost complex structure $J$. Then if $E$ is Courant involutive, i.e. closed under the Courant bracket (4.2), we say that $J$ is integrable and also a generalized complex structure. Note that $E$ is a maximal isotropic subbundle of $\left(T \oplus T^{*}\right) \otimes \mathbb{C}$.

As observed by P. Ševera and A. Weinstein [9], the Courant bracket (4.2) on $T \oplus T^{*}$ can be twisted by a real, closed 3-form $H$ on $\check{M}$ in the following way: given $H$ as above, define another important bracket $[\cdot, \cdot]_{H}$ on $T \oplus T^{*}$ by

$$
[X+\xi, Y+\eta]_{H}=[X+\xi, Y+\eta]+\iota_{Y} \iota_{X}(H),
$$

which is called $H$-twisted Courant bracket.

Definition 4.1. A generalized complex structure $J$ is said to be twisted generalized complex with respect to the closed 3 -form $H$ when its $+i$-eigenbundle $E$ is involutive with respect to the $H$-twisted Courant bracket and then the pair $(\check{M}, J)$ is called an $H$-twisted generalized complex manifold. 
From now on, we consider the $H$-twisted generalized complex manifold $(\check{M}, J)$ defined as above. We must remark that the following notations are not exactly the same as the usual ones since we just define them for our presentation below, and maybe miss their usual geometrical meaning. The twisted de Rham differential is given by

$$
d_{R}=d+(-1)^{k} R \wedge
$$

where $R \in \Omega^{k}(\check{M}, \mathbb{R})$. A natural action of $T \oplus T^{*}$ on smooth differential forms is given by

$(X+\xi) \cdot \alpha=\iota_{X}(\alpha)+\xi \wedge \alpha, \quad$ for any $X \in C^{\infty}(T), \xi \in C^{\infty}\left(T^{*}\right)$ and $\alpha \in \Omega^{*}(\check{M}, \mathbb{C})$.

Actually, this action can be considered as 'lowest level' of a hierarchy of actions on the bundles $T \bigoplus\left(\oplus_{r} \wedge^{r} T^{*}\right), r=1,2, \cdots$, defined by the similar formula

$$
\left(X+\xi_{1}+\xi_{2}+\cdots\right) \cdot \alpha=\iota_{X}(\alpha)+\xi_{1} \wedge \alpha+\xi_{2} \wedge \alpha+\cdots,
$$

for any $X \in C^{\infty}(T), \xi_{1}+\xi_{2}+\cdots \in C^{\infty}\left(\oplus_{r} \wedge^{r} T^{*}\right)$ and $\alpha \in \Omega^{*}(\check{M}, \mathbb{C})$. Then in the following discussion we adopt the action of $A=A_{1} \wedge \cdots \wedge A_{k} \in C^{\infty}\left(\wedge^{k}\left(T \bigoplus\left(\oplus_{r} \wedge^{r}\right.\right.\right.$ $\left.\left.T^{*}\right)\right)$ ) on $\Omega^{*}(\check{M}, \mathbb{C})$ given by

(4.3) $A \cdot \alpha=\left(A_{1} \wedge \cdots \wedge A_{k}\right) \cdot \alpha \equiv A_{1} \cdot A_{2} \cdots A_{k} \cdot \alpha, \quad$ for any $\alpha \in \Omega^{*}(\check{M}, \mathbb{C})$.

The generalized Schouten bracket for $A=A_{1} \wedge \cdots \wedge A_{p} \in C^{\infty}\left(\wedge^{p}\left(T \oplus T^{*}\right)\right)$ and $B=B_{1} \wedge \cdots \wedge B_{q} \in C^{\infty}\left(\wedge^{q}\left(T \oplus T^{*}\right)\right)$ is defined as

$$
[A, B]_{R}=\sum_{i, j}(-1)^{i+j}\left[A_{i}, B_{j}\right]_{R} \wedge A_{1} \wedge \cdots \wedge \hat{A}_{i} \wedge \cdots \wedge A_{p} \wedge B_{1} \wedge \cdots \wedge \hat{B}_{j} \wedge \cdots \wedge B_{q}
$$

where ^ means 'omission', the $R$-twisted Courant bracket $\left[A_{i}, B_{j}\right]_{R}$ is defined as $\left[A_{i}, B_{j}\right]+\iota_{Y_{j}} \iota_{X_{i}}(R)$ if we take $A_{i}=X_{i}+\xi_{i}$ and $B_{j}=Y_{j}+\eta_{j}$, and the action of $\left[A_{i}, B_{j}\right]_{R}$ comply with the principle of (4.3). Here we note that if $R$ is a 3 -form and $X+\xi, Y+\eta \in C^{\infty}\left(T \oplus T^{*}\right)$, then the $R$-twisted Courant bracket $[X+\xi, Y+\eta]_{R}$ still lie in $C^{\infty}\left(T \oplus T^{*}\right)$. However, for $R$ being general, the bracket $[X+\xi, Y+\eta]_{R}$ doesn't lie in $C^{\infty}\left(T \oplus T^{*}\right)$ in general since $\iota_{Y} \iota_{X}(R)$ is not necessarily a 1 -form, but in $C^{\infty}\left(T \bigoplus\left(\oplus \wedge^{*} T^{*}\right)\right)$; hence this bracket still makes sense under the action (4.3).

Proposition 4.2. (See also Lemma 4.24 of [3], (17) of [4] and Lemma 2 of [6].) For any smooth differential form $\rho$, any smooth odd-degree form $R$ and any $A \in C^{\infty}\left(\wedge^{p} E^{*}\right), B \in C^{\infty}\left(\wedge^{q} E^{*}\right)$, we have

$$
\begin{aligned}
d_{R}(A \cdot B \cdot \rho)= & (-1)^{p} A \cdot d_{R}(B \cdot \rho)+(-1)^{(p-1) q} B \cdot d_{R}(A \cdot \rho) \\
& +(-1)^{p-1}[A, B]_{R} \cdot \rho+(-1)^{p+q+1} A \cdot B \cdot d_{R} \rho .
\end{aligned}
$$

Proof. Firstly, we consider the initial case, i.e., $A, B \in C^{\infty}\left(E^{*}\right)$. It is proved by Gualtieri in Lemma 4.24 of [3] that

$$
A \cdot B \cdot d \rho=d(B \cdot A \cdot \rho)+B \cdot d(A \cdot \rho)-A \cdot d(B \cdot \rho)+[A, B] \cdot \rho-d\langle A, B\rangle \wedge \rho,
$$

where $A, B \in C^{\infty}\left(T \oplus T^{*}\right)$. Actually, (4.5) is essentially due to the commutator formula (2.3) and classical Cartan formula $L_{X}=d \circ \iota_{X}+\iota_{X} \circ d$. In our case, we can drop the last term involving the inner product. 
Later, Kapustin and Li proved the $H$-twisted version in (17) of [4] and then $\mathrm{Li}$ generalized it to any $A \in C^{\infty}\left(\wedge^{p} E^{*}\right)$ and $B \in C^{\infty}\left(\wedge^{q} E^{*}\right)$ in Lemma 2 of [6], where $H$ is a real closed 3 -form ${ }^{4}$. Here we give a slightly more general version when $R$ is any smooth form of odd degree. For the reader's convenience, we will write down the details though the essential idea of this process is due to [6].

Now let us compute $(A \cdot B) \cdot(R \wedge \rho)$. Let $A=X+\xi, B=Y+\eta$ and $R \in \Omega^{k}(\check{M}, \mathbb{R})$ with odd $k$. By a direct computation and the notations introduced above, we have the following two equalities

$$
\begin{aligned}
& B \cdot R \wedge(A \cdot \rho) \\
= & \left(\iota_{Y}+\eta \wedge\right)\left(R \wedge \iota_{X}(\rho)+R \wedge \xi \wedge \rho\right) \\
(4.6)= & \iota_{Y}\left(R \wedge \iota_{X}(\rho)\right)+\iota_{Y}(R \wedge \xi \wedge \rho)+\eta \wedge R \wedge \iota_{X}(\rho)+\eta \wedge R \wedge \xi \wedge \rho \\
= & \iota_{Y}(R) \wedge \iota_{X}(\rho)+(-1)^{k} R \wedge \iota_{Y} \iota_{X}(\rho)+\iota_{Y}(R) \wedge \xi \wedge \rho+(-1)^{k} R \wedge \iota_{Y}(\xi) \wedge \rho \\
& +(-1)^{k-1} R \wedge \xi \wedge \iota_{Y}(\rho)+\eta \wedge R \wedge \iota_{X}(\rho)+\eta \wedge R \wedge \xi \wedge \rho
\end{aligned}
$$

and

$$
B \cdot A \cdot \rho=\iota_{Y} \iota_{X}(\rho)+\iota_{Y}(\xi) \wedge \rho-\xi \wedge \iota_{Y}(\rho)+\eta \wedge \iota_{X}(\rho)+\eta \wedge \xi \wedge \rho .
$$

Hence, we have

$$
\begin{aligned}
& (A \cdot B) \cdot(R \wedge \rho) \\
= & \left(\iota_{X}+\xi \wedge\right)\left(\iota_{Y}(R \wedge \rho)+\eta \wedge R \wedge \rho\right) \\
= & \iota_{X} \iota_{Y}(R \wedge \rho)+\iota_{X}(\eta \wedge R \wedge \rho)+\xi \wedge \iota_{Y}(R \wedge \rho)+\xi \wedge \eta \wedge R \wedge \rho \\
= & \iota_{X} \iota_{Y}(R) \wedge \rho+(-1)^{k-1} \iota_{Y}(R) \wedge \iota_{X}(\rho)+(-1)^{k} \iota_{X}(R) \wedge \iota_{Y}(\rho)+R \wedge \iota_{X} \iota_{Y}(\rho) \\
& +\iota_{X}(\eta) \wedge R \wedge \rho-\eta \wedge \iota_{X}(R) \wedge \rho+(-1)^{k+1} \eta \wedge R \wedge \iota_{X}(\rho) \\
& +\xi \wedge \iota_{Y}(R) \wedge \rho+(-1)^{k} \xi \wedge R \wedge \iota_{Y}(\rho)+R \wedge \xi \wedge \eta \wedge \rho \\
= & R \wedge(B \cdot A \cdot \rho)+B \cdot R \wedge(A \cdot \rho)-A \cdot R \wedge(B \cdot \rho)-\iota_{Y} \iota_{X}(R) \wedge \rho,
\end{aligned}
$$

where the last equality applies the equalities (4.6) and (4.7). So by combining (4.5) with the last term dropped and (4.8) with minus sign, we complete the proof of the initial case of (4.4).

Then, by induction on the degrees of $A$ and $B$, we can conclude the proof. Actually, we just need to assume that (4.4) holds for all $p \leq r$ and $q=s$ and then show that it holds for $p=r+1$ and $q=s$ since (4.4) is graded symmetric in $A$ and $B$. Here we set $A=A_{0} \wedge \tilde{A}$ with $\tilde{A}=A_{1} \wedge \cdots \wedge A_{r}$ and $B=B_{1} \wedge \cdots \wedge B_{s}$, where all $A_{i}, B_{i} \in C^{\infty}\left(E^{*}\right)$. Assume that $A_{0}=X+\xi$, where $X \in C^{\infty}(T)$ and $\xi \in C^{\infty}\left(T^{*}\right)$.

\footnotetext{
${ }^{4}$ In [6], Yi Li proved an analog of the Bogomolov-Tian-Todorov theorem for $H$-twisted generalized Calabi-Yau manifolds by his critical Lemma 2, that is, the unobtruction and smoothness of the moduli space of generalized complex structures on a compact $H$-twisted generalized Calabi-Yau manifold.
} 
Then, one has

$$
\begin{aligned}
& d_{R}(A \cdot B \cdot \rho) \\
= & (d-R \wedge)\left(\iota_{X}+\xi \wedge\right)(\tilde{A} \cdot B \cdot \rho) \\
= & \left(L_{X}+d \xi \wedge \cdot-\iota_{X}(R) \wedge \cdot\right)(\tilde{A} \cdot B \cdot \rho)-A_{0} \cdot d_{R}(\tilde{A} \cdot B \cdot \rho) \\
= & \left(L_{X}+d \xi \wedge \cdot-\iota_{X}(R) \wedge \cdot\right)(\tilde{A} \cdot B \cdot \rho)-A_{0} \cdot\left((-1)^{r} \tilde{A} \cdot d_{R}(B \cdot \rho)\right. \\
& \left.+(-1)^{(r-1) s} B \cdot d_{R}(\tilde{A} \cdot \rho)+(-1)^{r-1}[\tilde{A}, B]_{R} \cdot \rho+(-1)^{r+s+1} \tilde{A} \cdot B \cdot d_{R} \rho\right) \\
= & (-1)^{(r+1)} A \cdot d_{R}(B \cdot \rho)+(-1)^{r s} B \cdot d_{R}(A \cdot \rho)+(-1)^{r+s} A \cdot B \cdot d_{R} \rho \\
& +(-1)^{r}\left(A_{0} \wedge[\tilde{A}, B]_{R}\right) \cdot \rho+\left(L_{X}+d \xi \wedge \cdot-\iota_{X}(R) \wedge \cdot\right)(\tilde{A} \cdot B \cdot \rho) \\
& -(-1)^{r s} B \cdot\left(L_{X}+d \xi \wedge \cdot-\iota_{X}(R) \wedge \cdot\right)(\tilde{A} \cdot \rho) .
\end{aligned}
$$

Claim 4.3. For any $C \in C^{\infty}\left(E^{*}\right)$ and $\alpha \in \Omega^{*}(\check{M}, \mathbb{C})$, we have

$$
\left[L_{X}+d \xi \wedge \cdot-\iota_{X}(R) \wedge \cdot C \cdot\right] \alpha=\left[A_{0}, C\right]_{R} \cdot \alpha,
$$

where the bracket $[\cdot, \cdot]$ on the left-hand side is just the usual Lie bracket.

Before the proof, we can easily see from this claim that the last two terms on the right-hand side of (4.9) combine to give us

$$
(-1)^{r s}\left(\left[A_{0}, B\right]_{R} \wedge \tilde{A}\right) \cdot \rho
$$

and then the last three terms on the right-hand side of (4.9) combine to give

$$
(-1)^{r}[A, B]_{R} \cdot \rho .
$$

Hence, one has

$$
\begin{aligned}
d_{R}(A \cdot B \cdot \rho)= & (-1)^{(r+1)} A \cdot d_{R}(B \cdot \rho)+(-1)^{r s} B \cdot d_{R}(A \cdot \rho) \\
& +(-1)^{r+s} A \cdot B \cdot d_{R} \rho+(-1)^{r}[A, B]_{R} \cdot \rho,
\end{aligned}
$$

by which we complete the induction.

Finally, we prove Claim 4.3 to conclude the proof. If we write $C=Y+\eta$, then

$$
\begin{aligned}
& {\left[L_{X}+d \xi \wedge \cdot-\iota_{X}(R) \wedge \cdot C \cdot\right] \alpha } \\
= & L_{X} \iota_{Y}(\alpha)-\iota_{Y}\left(L_{X} \alpha\right)+\left(L_{X} \eta\right) \wedge \alpha+\iota_{Y} \iota_{X}(R) \wedge \alpha-\iota_{Y}(d \xi) \wedge \alpha \\
= & \iota_{[X, Y]}(\alpha)+\left(L_{X} \eta\right) \wedge \alpha+\iota_{Y} \iota_{X}(R) \wedge \alpha-\iota_{Y}(d \xi) \wedge \alpha \\
= & \iota_{[X, Y]}(\alpha)+\left(L_{X} \eta\right) \wedge \alpha-\left(L_{Y} \xi\right) \wedge \alpha-\frac{1}{2} d\left(\iota_{X}(\eta)-\iota_{Y}(\xi)\right) \wedge \alpha+\iota_{Y} \iota_{X}(R) \wedge \alpha \\
= & {[X+\xi, Y+\eta] \cdot \alpha+\iota_{Y} \iota_{X}(R) \wedge \alpha } \\
= & {\left[A_{0}, C\right]_{R} \cdot \alpha, }
\end{aligned}
$$

where the second equality uses the commutator formula (2.3): $L_{X} \circ \iota_{Y}-\iota_{Y} \circ L_{X}=$ $\iota_{[X, Y]}$, and the third equality uses the fact that $\iota_{X}(\eta)+\iota_{Y}(\xi)=0$ and also the classical Cartan formula $L_{X}=d \circ \iota_{X}+\iota_{X} \circ d$.

COROLlaRY 4.4. For any smooth differential form $\rho$, any smooth 1 -form $R$ and any $A, B \in C^{\infty}\left(\wedge^{2} E^{*}\right)$, we have

$$
d_{R}(A \cdot B \cdot \rho)=A \cdot d_{R}(B \cdot \rho)+B \cdot d_{R}(A \cdot \rho)-[A, B] \cdot \rho-A \cdot B \cdot d_{R} \rho .
$$


Obviously, similar to (3.1) vs (3.2), we can obtain an equivalent form of (4.10).

Then, we can reprove Corollary 3.4 for any Hermitian holomorphic vector bundle on a complex manifold.

COROLlary 4.5. Let $V$ be an arbitrary Hermitian holomorphic vector bundle on the complex manifold $M$. For any $\omega \in A^{*, *}(V)$ and any $\phi_{i} \in A^{0,1}\left(M, T_{M}^{1,0}\right), i=1,2$, there holds

$$
\left.\left.\left.\left.\left.\left.\left.\left.\left.\left[\phi_{1}, \phi_{2}\right]\right\lrcorner \omega=\phi_{1}\right\lrcorner \nabla\left(\phi_{2}\right\lrcorner \omega\right)-\nabla\left(\phi_{2}\right\lrcorner\left(\phi_{1}\right\lrcorner \omega\right)\right)+\phi_{2}\right\lrcorner \nabla\left(\phi_{1}\right\lrcorner \omega\right)-\phi_{2}\right\lrcorner\left(\phi_{1}\right\lrcorner \nabla \omega\right),
$$

where $\nabla$ is the Chern connection of the Hermitian holomorphic vector bundle $V$.

Proof. This corollary is a direct application of Corollary 4.4 when we set $A=$ $\left(\phi_{1}\right)^{i} \cdot \partial_{i}$ and $B=\left(\phi_{2}\right)^{j} \cdot \partial_{j}$ and take $R$ as the connection $(1,0)$-form matrix $\theta$ of the connection $\nabla$ with respect to a holomorphic frame $s$ of $V$ with minus sign, by the same principle as we choose $g \cdot \partial f$ in the proof of Theorem 3.3. It is obvious that $E$ in Corollary 4.4 is taken as $T^{0,1} \otimes T^{* 1,0}$ in our case. More precisely, since

$$
\begin{aligned}
{[A, B] } & =\left[\left(\phi_{1}\right)^{i} \cdot \partial_{i},\left(\phi_{2}\right)^{j} \cdot \partial_{j}\right] \\
& =-\left[\left(\phi_{1}\right)^{i}, \partial_{j}\right] \wedge \partial_{i} \wedge\left(\phi_{2}\right)^{j}-\left[\partial_{i},\left(\phi_{2}\right)^{j}\right] \wedge\left(\phi_{1}\right)^{i} \wedge \partial_{j} \\
& =\iota \partial_{j}\left(d\left(\phi_{1}\right)^{i}\right) \wedge \partial_{i} \wedge\left(\phi_{2}\right)^{j}-\iota \partial_{i}\left(d\left(\phi_{1}\right)^{j}\right) \wedge\left(\phi_{1}\right)^{i} \wedge \partial_{j} \\
& =\left(\phi_{2}\right)^{j} \wedge \partial_{j}\left(\phi_{1}\right)^{i} \wedge \partial_{i}+\left(\phi_{1}\right)^{i} \wedge \partial_{j}\left(\phi_{2}\right)^{j} \wedge \partial_{j},
\end{aligned}
$$

then one has

$$
\left.[A, B] \cdot \omega=\left[\phi_{1}, \phi_{2}\right]\right\lrcorner \omega .
$$

Moreover, one easily knows that

$$
\begin{gathered}
\left.\left.A \cdot d_{R}(B \cdot \omega)=\phi_{1}\right\lrcorner \nabla\left(\phi_{2}\right\lrcorner \omega\right), \\
\left.\left.B \cdot d_{R}(A \cdot \omega)=\phi_{2}\right\lrcorner \nabla\left(\phi_{1}\right\lrcorner \omega\right), \\
\left.\left.d_{R}(A \cdot B \cdot \omega)=\nabla\left(\phi_{2}\right\lrcorner\left(\phi_{1}\right\lrcorner \omega\right)\right)
\end{gathered}
$$

and

$$
\left.\left.A \cdot B \cdot d_{R} \omega=\phi_{2}\right\lrcorner\left(\phi_{1}\right\lrcorner \nabla \omega\right) .
$$

Hence, by substituting the five equalities above into $(4.10) \otimes s$ the equivalent form of (4.10), we complete our proof.

Almost by the same argument as the previous corollary, we can generalize it to any polyvector fields as follows.

COROLlary 4.6. Let $V$ be an arbitrary Hermitian holomorphic vector bundle on the complex manifold $M$. For any $\omega \in A^{*, *}(V)$ and any $\phi_{i} \in A^{0, q_{i}}\left(M, \bigwedge^{p_{i}} T_{M}^{1,0}\right)$, $i=1,2$, there holds

$$
\left.\left.\left.\left.\left.\left.\left.\left.\left.\left[\phi_{1}, \phi_{2}\right]\right\lrcorner \omega=\phi_{1}\right\lrcorner \nabla\left(\phi_{2}\right\lrcorner \omega\right)-\nabla\left(\phi_{2}\right\lrcorner\left(\phi_{1}\right\lrcorner \omega\right)\right)+\phi_{2}\right\lrcorner \nabla\left(\phi_{1}\right\lrcorner \omega\right)-\phi_{2}\right\lrcorner\left(\phi_{1}\right\lrcorner \nabla \omega\right),
$$

where $\nabla$ is the Chern connection of the Hermitian holomorphic vector bundle $V$ and $[\cdot, \cdot]$ on the LHS is the standard Schouten-Nijenhuys bracket on polyvector fields. 
Acknowledgement. The second author would like to thank Professor A. Todorov, Dr. Fangliang Yin and Shengmao Zhu for several useful talks, and specially Dr. Feng Guan for his constructive suggestion on Theorem 3.3 during his visit to Center of Mathematical Sciences, Zhejiang University. Moreover, we also would like to thank the referee for their useful and accurate comments improving our presentation a lot.

\section{REFERENCES}

[1] S. Barannikov and M. Kontsevich, Frobenius manifolds and formality of Lie algebras of polyvector fields, Internat. Math. Res. Notices, 4 (1998), pp. 201-215.

[2] R. FRIEDman, On threefolds with trivial canonical bundle, Complex geometry and Lie theory (Sundance, UT, 1989), pp. 103-134, Proc. Sympos. Pure Math., 53, Amer. Math. Soc., Providence, RI, 1991.

[3] M. Gualtieri, Generalized Complex Geometry, D. Phil thesis, Oxford University, arXiv:math.DG/0401221.

[4] A. Kapustin And Y. LI, Topological sigma-models with $H$-flux and twisted generalized complex manifolds, Adv. Theor. Math. Phys., 11:2 (2007), pp. 269-290.

[5] S. LAng, Differential and Riemannian manifolds, Third edition, Graduate Texts in Mathematics, 160, Springer-Verlag, New York, 1995.

[6] Y. LI, On deformations of generalized complex structures the generalized Calabi-Yau case, arXiv:hep-th/0508030v2 15 Oct 2005.

[7] K. LiU, X. Sun And S.-T. YAU, Recent development on the geometry of the Teichmüller and moduli spaces of Riemann surfaces, Surveys in Differential Geometry XIV, 2009, pp. 221259.

[8] J. Morrow And K. Kodaira, Complex manifolds, Holt, Rinehart and Winston, Inc., New York-Montreal, Que.-London, 1971.

[9] P. Ševera And A. Weinstein, Poisson geometry with a 3-form background, Prog. Theor. Phys. Suppl., 144 (2001), pp. 145-154.

[10] G. TIAN, Smoothness of the universal deformation space of Calabi-Yau manifolds and its Petersson-Weil metric, Math. Aspects of String Theory, ed. S.-T.Yau, World Scientic (1986), pp. 629-646.

[11] A. Todorov, The Weil-Petersson geometry of moduli spaces of $\mathbb{S U}(n \geq 3)$ (Calabi-Yau manifolds) I, Comm. Math. Phys., 126 (1989), pp. 325-346.

[12] A. Todorov, Moduli space of polarized CY manifolds, preprint. 
K. LIU AND S. RAO 